Open Access

Original Article

\title{
Correlations of CT scan with high-sensitivity C-reactive protein and D-dimer in patients with coronavirus disease 2019
}

\author{
Jin Zhu', Cheng Chen ${ }^{2}$, Rongshu Shi ${ }^{3}$, Bangguo $\mathrm{Li}^{4}$
}

\begin{abstract}
Objectives: To study the correlations of CT scan with high-sensitivity C-reactive protein (hs-CRP) and D-dimer in patients with coronavirus disease 2019 (COVID-2019).

Methods: From January to March 2020, COVID-19 patients were divided into two groups according to the Diagnosis and Treatment Protocol for Novel Coronavirus Pneumonia (trial version 7), with mild and ordinary cases as Group-1 and critical and severe cases as Group-2. The chest CT scan results, hs-CRP, D-dimmer levels of the two groups from admission to discharge were compared by the $c^{2}$ test or Fisher's exact test. The quantitative data were represented as mean \pm standard deviation $(\bar{x} \pm s)$. Intergroup comparisons were performed by the independent samples $t$ test, and the ineligible data were subjected to the nonparametric rank sum test. Binary logistic regression model was used for multivariate correlation analysis, using independent variables that were significant in univariate analysis. The correlations between the above indices were analyzed.

Results: In Group-1, there were two cases of normal chest CT scan results, one case of fibrosis, and 25 cases of abnormalities during the first diagnosis, mainly manifested as single or scattered ground-glass shadows. After treatment, the CT scan results became normal. The chest CT scan of Group-2 showed abnormalities, including 21 cases of multiple ground-glass shadows, and six cases of multiple consolidations accompanied by ground-glass shadows, who were critically ill and died. In addition, there were 16 cases of multiple ground glass shadows with partial consolidation, and the CRP and D-dimer levels of Group-2 were significantly higher than those of Group-1. Chest CT scan results were significantly positively correlated with CRP and $D$-dimer levels $(P<0.05)$.

Conclusion: The chest CT scan results of COVID-19 patients are characteristic, being correlated with CRP and D-dimer levels. D-dimer and CRP levels significantly increase in most severe and critical patients, which are closely related to their prognosis. The indices may play predictive roles in clinical treatment and prognosis evaluation.
\end{abstract}

KEYWORDS: Coronavirus disease 2019; Pulmonary fibrosis; D-dimer; High-sensitivity C-reactive protein.

How to cite this:

doi: https://doi.org/10.12669/pjms.36.6.2961

Zhu J, Chen C, Shi R, Li B. Correlations of CT scan with high-sensitivity C-reactive protein and D-dimer in patients with coronavirus disease 2019. Pak J Med Sci. 2020;36(6):1397-1401. doi: https://doi.org/10.12669/pjms.36.6.2961

This is an Open Access article distributed under the terms of the Creative Commons Attribution License (http://creativecommons.org/licenses/by/3.0), which permits unrestricted use, distribution, and reproduction in any medium, provided the original work is properly cited.

\section{INTRODUCTION}

Correspondence:

Bangguo Li

Affiliated Hospital of Zunyi Medical University,

Zunyi 563003, P. R. China.

Email: writslimhyd@web.de

* Received for Publication:

* Accepted for Publication:

May 26, 2020

August 3, 2020
In December 2019, patients with Coronavirus Disease 2019 (COVID-19) were first identified and diagnosed in Wuhan, Hubei province. ${ }^{1}$ The International Committee on Taxonomy of Viruses officially named the virus that caused the disease as severe acute respiratory syndrome coronavirus 2 on February 10th, 2020.,.$^{2,3}$ The main clinical 
manifestations of COVID-19 patients mainly included fever, dry cough and fatigue, and the diagnosis was mainly based on the positivity of coronavirus nucleic acid detected by reverse transcription-polymerase chain reaction (RT-PCR) for the respiratory samples of suspected patients with epidemiological history and corresponding symptoms. ${ }^{1}$

CT scores and serum high-sensitivity C-reactive protein (hs-CRP) level have good consistency, and the combination of them can dynamically and effectively assess disease progression and therapeutic effects. ${ }^{4} \mathrm{Hs}$-CRP is an inflammatory response protein, the increase of which in serum can reflect the activation of inflammatory response. ${ }^{5}$ In patients with thrombosis, the increase in its serum level initiates the coagulation cascade reaction, and accelerates the formation of thrombi by activating the complement system and initiating the exogenous coagulation pathway. ${ }^{6}$ The systemic anatomy of a dead case of COVID-19 showed that there was microthrombosis in the pulmonary artery. ${ }^{7}$ The thrombosis of pulmonary arterioles in patients with severe COVID-19 can aggravate the progression of pulmonary lesions or delay the absorption of lesions.

In patients recovering from COVID-19 (without severe respiratory distress during the disease course), the lung abnormalities disclosed by chest CT scan became most severe about 10 days after the initial symptoms appeared. ${ }^{8}$ In this study, the correlations of chest CT scan results with hs-CRP and D-dimer levels in 71 mild and severe cases upon the first diagnosis were analyzed. The severity of chest CT lesions was positively correlated with hs-CRP and D-dimer levels which significantly increased in the patients with severe COVID-19. According to the pharmaceutical monitoring of the prevention and treatment for COVID-19-related circulatory system thrombosis, ${ }^{9}$ the clinical monitoring of the above indices can evaluate the prognosis and therapeutic efficacy.

Seventy-one COVID-19 patients diagnosed in Ezhou Central Hospital during the medical aid to Hubei province from January 3rd to February 26th, 2020 were retrospectively analyzed, all of whom were hospitalized after being detected positive by RT-PCR. According to the Diagnosis and Treatment Protocol for Novel Coronavirus Pneumonia (trial version 7), ${ }^{1}$ the patients with COVID-19 were divided into two groups, with mild and ordinary cases as Group- $1(n=28)$ and critical and severe cases as Group-2 $(n=43)$. In Group-1, there were 18 males and 10 females aged 9-84 years old, with an average of (15.971 \pm 49.607$)$. In Group-2, there were 26 males and 17 females aged 35-94 years, with an average of (16.674 \pm $60.884)$. The chest CT foci as well as changes of hs-CRP and D-dimer levels from admission to discharge were recorded.

With the patient breathing calmly, CT scan was conducted from the entrance of the thorax to the lower part of the diaphragm. Scan parameters: Tube voltage, $120 \mathrm{kV}$; tube current, $320 \mathrm{~mA}$; scan pitch, 0.875; slice thickness, $5 \mathrm{~mm}$; slice spacing, $5 \mathrm{~mm}$; matrix, $512 \times 512$. All CT images were obtained through lung window and mediastinal window.

In the early morning, $3-5 \mathrm{ml}$ of fasting venous blood was drawn from each patient, and the serum was collected after centrifugation to measure hs-CRP and D-dimer levels using an automatic biochemical analyzer. Blood collection and CT scan were carried out on the same day.

All data were statistically analyzed by SPSS 21.0 software. The numerical data were expressed as case number (\%). Intergroup comparisons were performed by the $c^{2}$ test or Fisher's exact test. The quantitative data were represented as mean \pm standard deviation $(\bar{x} \pm s)$. Intergroup comparisons were conducted by the independent samples $t$ test, and the ineligible data were subjected to the nonparametric rank sum test. Binary logistic regression model was used for multivariate correlation analysis, using independent variables that were significant in univariate analysis. The results were expressed as corrected odds ratio (OR) and corresponding 95\% confidential interval $(\mathrm{CI}) . \mathrm{P}<0.05$ was considered statistically significant.

\section{RESULTS}

CT scan results: In Group-1, there were two cases of normal chest CT scan results, one case of fibrosis, and 25 cases of abnormalities during the first diagnosis, mainly manifested as single or scattered ground-glass shadows (Fig.1). After treatment, the CT scan results became normal (Fig.2). The chest CT scan of Group-2 showed abnormalities, including 21 cases of multiple ground-glass shadows, and six cases of multiple consolidations accompanied by ground-glass shadows, who were critically ill and died. Additionally, there were 16 cases of multiple ground glass shadows with partial consolidation. 


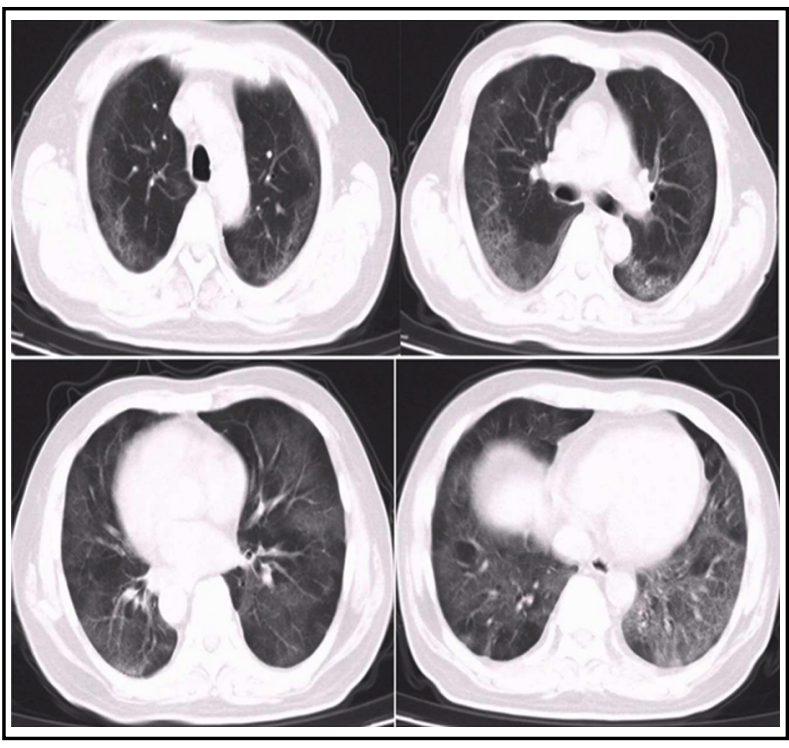

Fig. 1: Chest CT scan results of a 53-year-old COVID-19 patient admitted after fever for 4 days. At admission, there were multiple ground-glass changes in both lungs. D-dimer level was $5.56 \mu \mathrm{g} / \mathrm{ml}$, and hs-CRP level was $22.2 \mathrm{mg} / \mathrm{L}$. He was given antiviral, immunityenhancing and anticoagulant therapies.

Laboratory examination results: The hs-CRP levels of most cases significantly increased (53 cases), and those of a few were normal (18 cases). Such levels of 19 cases (26.7\%) in Group-1 were elevated, and those of 34 cases $(47.8 \%)$ in Group-2 were raised. The D-dimer levels of 45 cases were significantly elevated, and those of 26 cases were normal. Only one case was elevated and the rest were normal in Group-1. In Group-2, such levels of 44 cases were raised, accounting for $61.9 \%$.

Correlation analysis: The levels of hs-CRP and D-dimer in Group-2 significantly exceeded those

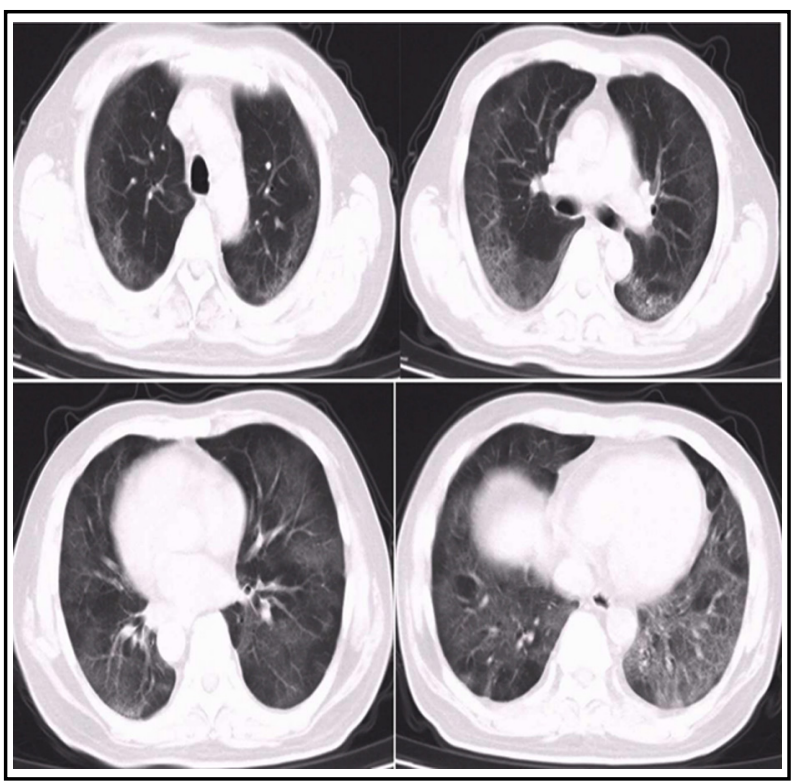

Fig. 2: Chest CT scan results after 15 days of hospitalization. Multiple ground-glass lesions were observed, and pulmonary fibrosis was found locally. $\mathrm{D}$-dimer and hs-CRP levels reduced to $0.62 \mu \mathrm{g} / \mathrm{ml}$ and $14.6 \mathrm{mg} / \mathrm{L}$, respectively.

of Group-1 (Table-I). There were no significant differences in other indices between the two groups $(\mathrm{P}>0.05)$. The logistic regression analysis showed that there was a significant positive correlation between D-dimer level and severe symptoms $(\mathrm{P}<0.05)($ Table-II), so this level was an independent risk factor $(\mathrm{OR}>1)$ that affected the outcome. The probability of severe outcome rose 1.460-fold (OR: 2.460) with every unit increase of D-dimer level. There were significant positive correlations between chest $\mathrm{CT}$ scan results and levels of hs-CRP and D-dimer $(r>0$, $\mathrm{P}<0.05)$.Table-III.

Table-I: Baseline clinical data $[\mathrm{n}(\%),(\bar{x} \times \pm \mathrm{s})]$.

\begin{tabular}{lcccc}
\hline Index & Group-1 $(n=28)$ & Group-2 $(n=43)$ & $\chi^{2} / t / Z$ & $P$ \\
\hline hs-CRP & $10.048 \pm 8.860$ & $18.236 \pm 7.026$ & $-3.059^{\Delta}$ & 0.002 \\
D-dimer & $0.965 \pm 0.854$ & $3.273 \pm 7.053$ & $-3.765^{\Delta}$ & 0.000 \\
\hline
\end{tabular}

*: Chi-square test; ${ }^{\#}$ : $t$ test; ${ }^{\Delta}$ : Z test; ${ }^{\nabla}$ : Fisher's exact test.

Table-II: Logistic regression analysis results of severe cases.

\begin{tabular}{lccccccc}
\hline Item & $B$ & $S E$ & Wald & OR & \multicolumn{2}{c}{$95 \% C I$} & $P$ \\
\cline { 5 - 7 } & & & & & Lower limit & Upper limit & \\
\hline hs-CRP & 0.086 & 0.044 & 3.796 & 1.089 & 0.999 & 1.188 & 0.051 \\
D-dimer & 0.900 & 0.432 & 4.336 & 2.460 & 1.054 & 5.742 & 0.037 \\
\hline
\end{tabular}

B: Coefficient estimate; Wald: chi-square value;

OR: odds ratio which represents the unit amount of experimental variable increase; CI: confidential interval. 
Table-III: Correlations of CT scan results with levels of CRP and D-dimer.

\begin{tabular}{lcc}
\hline Index & $r$ & $P$ \\
\hline CRP & 0.237 & 0.046 \\
D-dimer & 0.288 & 0.015 \\
\hline
\end{tabular}

\section{DISCUSSION}

COVID-19 belongs to the novel coronavirus of the genus $\beta$, with an envelope; the particles are round or oval in shape, which are usually polymorphic, with a diameter of 60 to $140 \mathrm{~nm}$. It is known that the virus is highly contagious, mainly transmitted through respiratory tract and contact. The virus invades the interior of the cell by completing receptor binding with angiotensin-converting enzyme II of mucosal cells. ${ }^{10}$ Serum hs-CRP levels and CT findings of severe patients were consistent with the newly reported pathological manifestations, which were diffuse alveolar lesion under microscope, a large amount of exudative monocytes, lymphocytes and plasma cells in the alveolar cavity and pulmonary interstitium, and extensive pulmonary interstitial fibrosis. ${ }^{11}$ Deng et al. studied the correlation between CT scores and hs-CRP levels at different stages of COVID-19 progression, and obtained a good consistency between CT scores and serum hs-CRP values. ${ }^{12}$ The combination of the two indicators can be used to dynamically and effectively evaluate the changes in the conditions, as well as the efficacy.

D-dimer is one of the specific products of crosslinked fibrin and one of the hallmarks of secondary fibrinolysis in vivo. The increased D-dimer content reflects the enhanced activity of secondary fibrinolysis, and the sensitivity of D-dimer in the diagnosis of thrombosis is $95 \%$, so the detection of plasma D-dimer is highly sensitive to the diagnosis of acute thrombosis, which has been widely used in the screening of thrombosis abroad. ${ }^{13} \mathrm{Hs}-\mathrm{CRP}$ is a cell membrane glycoprotein that stimulates and induces monocytes to express tissue factor which is a key factor that initiates the body's coagulation waterfall reaction to produce thrombosis, and at the same time, hs-CRP also activates the complement system, and damages vascular intima, thus promoting thrombosis. ${ }^{14}$ The serum hs-CRP levels and CT findings of severe patients were consistent with the newly reported pathological manifestations, which were diffuse alveolar lesion under microscope, a large amount of exudative monocytes, lymphocytes and plasma cells in the alveolar cavity and pulmonary interstitium, and extensive pulmonary interstitial fibrosis. ${ }^{11}$ In this study, 71 patients were divided into mild and severe patients, and the chest CT, hs-CRP, and D-dimer levels were dynamically monitored. In mild patients, early pulmonary lesions are of light density, mainly in the form of cloud and ground glass nodules, with limited scope. ${ }^{15}$ After treatment, pulmonary lesions could be obviously absorbed, even without residual fibrosis. In severe patients, as the disease progressed, the number and density of lesions increased, the scope expanded, and multiple lobes were involved, ${ }^{16}$ and obvious pulmonary fibrosis remained after treatment. In the course of treatment, the hsCRP and D-dimer levels in severe patients were significantly higher than those in mild patients, showing a progressive increase. After the disease was effectively controlled, most of the young severe patients with good physical fitness had a significantly improved absorption of pulmonary lesions, significantly reduced CT manifestations, decreased density and more common pulmonary fibrosis, the pulmonary lesions of some patients were completely absorbed, ${ }^{17}$ and the levels of hsCRP and D-dimer fell to the normal range. If the disease continued to progress, it might develop into a critical stage, the CT results showed diffuse consolidation of both lungs, with the manifestations of white lung. ${ }^{18}$ In some patients, the hs-CRP and D-dimer levels continued to rise, the inflammatory response of the body aggravated, complicated with sepsis, leading to systemic multiple organ failure. We found that the hs-CRP and D-dimer levels were significantly increased in severe patients, and the level of increase was consistent with the aggravation degree of chest CT lesions. According to the systemic anatomy of a dead COVID-19 patient, microthrombosis existed in the pulmonary artery. ${ }^{7}$ Therefore, for COVID-19 patients, the D-dimer level should also be closely detected, and routine anticoagulation should be performed when there are no contraindications.

\section{CONCLUSION}

Many studies have reported that chest CT progress is positively correlated with elevated hsCRP levels, which was also found in this study. However, the hs-CRP level is closely related to the occurrence of thrombosis, and D-dimer levels in critical patients continue to increase. Chest CT scan, hs-CRP and D-dimer levels have good consistency. 
In the clinical process of diagnosis and treatment, if the scope of chest CT lesions is expanded and the disease progresses rapidly, hs-CRP and D-dimer levels can be closely monitored. If anticoagulant therapy is given without contraindications, the conditions of most patients can be significantly alleviated, and dynamic and effective evaluation of the condition changes can be performed in combination with several indicators, and the therapeutic effect can also be evaluated, so as to help timely adjust the diagnosis and treatment plan in clinical practice.

\section{Source of funding: None.}

Conflict of Interest: None to declare.

\section{REFERENCES}

1. National Health Commission \& State Administration of Traditional Chinese Medicine. Diagnosis and Treatment Protocol for Novel Coronavirus Pneumonia (Trial Version 7). Available at: http://www. nhc.gov.cn/yzygj/s7653p/ 202003/46c9294a7dfe4cef80dc7f5912eb 1989.shtml.

2. Zhu N, Zhang D, Wang W, Li X, Yang B, Song J, et al. A novel coronavirus from patients with pneumonia in China, 2019. N Engl J Med. 2020;382(8):727-733. doi: 10.1056/ NEJMoa2001017

3. Gorbalenya AE. Severe acute respiratory syndromerelated coronavirus: The species and its viruses-a statement of the Coronavirus Study Group. BioRxiv. 2020. doi: 10.1101/2020.02.07.937862

4. Kim SH, Lee JY, Kim DH, Ham JH, Song YK, Lim EJ, et al. Factors related to the initial stroke severity of posterior circulation ischemic stroke. Cerebrovasc Dis. 2013;36(1):6268. doi: $10.1159 / 000351512$

5. Falanga A, Russo L, Verzeroli C. Mechanisms of thrombosis in cancer. Thromb Res. 2013;131 Suppl 1:S5962. doi: 10.1016/S0049-3848(13)70024-0

6. Entezari-Maleki T, Talasaz AH, Salarifar M, Hadjibabaie M, Javadi MR, Bozorgi A, et al. Plasma Vitamin D Status and Its Correlation with Risk Factors of Thrombosis, P-selectin and hs-CRP Level in Patients with Venous Thromboembolism; The First Study of Iranian Population. Iran J Pharm Res. 2014;13(1):319-327.

7. Liu Q, Wang RS, Qu GQ, Wang YY, Liu P, Zhu YZ, et al. [Observation Report on Systemic Anatomy of Dead Cases of Novel Coronavirus 2019 Pneumonia]. J Forensic Med. 2020;36(1):21-23.

8. Pan F, Ye T, Sun P, Gui S, Liang B, Li L, et al. Time Course of Lung Changes on Chest CT During Recovery From 2019 Novel Coronavirus (COVID-19) Pneumonia. Radiology. 2020;295(3):715-721. doi: 10.1148/radiol.2020200370

9. Xiong $Y$, Jiang $M$, Bian $Y$, Ling $W$, Tong RS, Yan JF, et al. [Pharmacy monitoring of prevention and treatment of thrombosis associated with novel coronavirus 2019 pneumonia]. West China J Pharm Sci. available at: http:/ / kns.cnki.net/kcms/detail/51.1218.R.20200310.1829.002. html.
10. Huang C, Wang Y, Li X, Ren L, Zhao J, Hu Y, et al. Clinical features of patients infected with 2019 novel Coronavirus in Wuhan, China. Lancet. 2020;395(10223):497-506. doi: 10.1016/S0140-6736(20)30183-5

11. Luo W, Yu H, Gou J, Li X, Sun Y, Li J, et al. Clinical Pathology of Critical Patient with Novel Coronavirus Pneumonia (COVID-19). Preprints. 2020;2020:2020020407.

12. Deng LT, Zou YY, Wang Y, Deng QC, Tian RH. [Correlation between $\mathrm{CT}$ scores and high-sensitivity $\mathrm{C}$-reactive protein at different progression stages of novel coronavirus 2019 pneumonia]. Acad J Chin PLA Med School. Available at: https://kns8.cnki.net/KCMS/detail/10.1117.r.20200330.1 034.014.html.(Cited May 5, 2020)

13. Linkins LA, Bates SM, Lang E, Kahn SR, Douketis JD, Julian J, et al. Selective D-Dimer Testing for Diagnosis of a First Suspected Episode of Deep Venous Thrombosis: A Randomized Trial. Ann Intern Med. 2013;158(2):93-100. doi: 10.7326/0003-4819-158-2-201301150-00003

14. Shrivastava AK, Singh HV, Raizada A, Singh SK. C-reactive protein, inflammation and coronary heart disease. Egypt Heart J. 2015;67(2):89-97. doi: 10.1016/j.ehj.2014.11.005.

15. Guan HX, Xiong Y, Shen NQ, Fan YQ, Shao JB, Li HJ, et al. [Clinical imaging features of novel coronavirus 2019 (COVID-19) pneumonia]. Radiol Pract. 2020;35(2):125-130. doi: 10.13609/j.cnki.1000-0313.2020.02.001

16. Liu FM, Ding HL, Gong XM, Song L, Li H, Lu ZY. [Chest CT manifestations and clinical features of novel coronavirus 2019 (COVID-19) pneumonia]. Radiol Pract. 2020;35(3):266268. doi: 10.13609/j.cnki.1000-0313.2020.03.004

17. Wang JC, Liu JP, Wang YY, Liu W, Chen XQ, Sun C, et al. [Chest CT imaging dynamic changes of patients with novel coronavirus 2019 (COVID-19) pneumonia]. J Zhejiang Univ (Med Sci). 2020;49(2):191-197.

18. Ji GH, Huang $\mathrm{MH}$, Zhang $\mathrm{Q}$, Wang WB, Wang $\mathrm{P}$, Qin $\mathrm{XT}$, et al. [CT findings and dynamic changes of novel coronavirus 2019 pneumonia]. Chin J Med Imaging Technol. doi: 10.13929/j.issn.1003-3289.2020.02.01

\section{Authors' Contributions:}

JZ \& BL: Study design and significant manuscript revision.

CC \& RS: Manuscript drafting, clinical data collection and analysis.

JZ, CC, RS \& BL: Approval of manuscript submission.

Authors:

1. Jin Zhu,

Department of Intervention,

2. Cheng Chen,

Department of Thoracic Surgery,

3. Rongshu Shi,

Department of Intervention,

4. Bangguo $\mathrm{Li}$,

Department of Imaging,

1-4: Affiliated Hospital of Zunyi Medical University, Zunyi 563003, P.R. China. 